\title{
Comparison of albumin, total protein, globulin and enzyme activity Creatine Phospho Kinase (CPK) in the Gingival Crevicular Fluid of patients with chronic periodontitis before and after treatment
}

\author{
Ali Banihashem Rad $^{1 *}$, Shade Saghafi ${ }^{2}$, Eshagh Hashemi ${ }^{3}$, Ershad Aghasizadeh ${ }^{4}$, Fathollah Ebrahimi ${ }^{5}$, Ahmad \\ Banihashem $^{6}$ and Sara Rajaei ${ }^{7}$ \\ ${ }^{1}$ Associated professor, Department of Periodontics, School of Dentistry and Dental Researches Center of Mashhad University of Medical Sciences, \\ Mashhad, Iran \\ ${ }^{2}$ Associated professor, Department of Oral and Maxillofacial Pathology, Oral and Maxillofacial Diseases Research Center, School of Dentistry and \\ ${ }^{3}$ Assistant professor, Mashad University of Medical Sciences \\ ${ }^{4}$ Periodontist, N0 $174^{\text {th }}$ Aref Street Mashhad Iran \\ ${ }^{5}$ General Dentist, School of Dentistry, Vakilabad BLVD, Mashad Iran \\ ${ }^{6}$ Student of Dentistry, School of Dentistry, Vakilabad Blvd, Mashad Iran \\ ${ }^{7}$ Pedodontist, N0 $174^{\text {th }}$ Aref Street Mashhad Iran
}

Received 10 Aug 2018, Accepted 12 Oct 2018, Available online 15 Oct 2018, Vol.6 (Sept/Oct 2018 issue)

\begin{abstract}
Introduction: Periodontal disease is an inflammatory disease of the supporting tissues of the teeth caused by certain microorganisms and leads to progressive destruction of the periodontal ligament, alveolar bone with pocket formation or the recession, or both. Periodontal disease is usually based on clinical parameters diagnosed and recorded. Other advanced diagnostic techniques of periodontal disease is evaluation of host response, which includes the study of specific or non-specific mediators such as albumin, globulin and creatine phosphokinase enzyme by biochemical methods or immunological response that as part of the individual periodontal infections are known.

Methods: The study population consisted of 30 patients. After observing isolation by cotton rolls, 50 to $100 \mathrm{ml} \mathrm{GCF}$ via 30 paper point and wetting with a height of $5 \mathrm{~mm}$ of the area with more involvement in terms of standard of patients with moderate periodontitis referred to Periodontics ward of Dental School in Mashhad pre-treatment phase 1 (scaling \& root planning) and 4 weeks after the treatment is provided. Then changes in albumin, total protein, globulin and CPK enzyme activity in the GCF using biochemical kits measured.

Result: In our study, mean CPK in patients with chronic periodontitis before treatment was further after treatment but not significantly different, but the average amount of albumin, total protein and globulin chronic periodontitis patients before treatment lesser than after treatment but there was no significant difference either.

Conclusion: In this study, we showed that albumin, total protein and globulin was significantly increased after treatment of periodontitis patients.
\end{abstract}

Keywords: GCF, periodontitis, albumin, creatine phosphokinase

Introduction

Periodontal disease is an inflammatory disease of the supporting tissues of the teeth caused by certain microorganisms and leads to progressive destruction of the periodontal ligament, alveolar bone with pocket formation or the recession, or both. Periodontal disease is usually based on clinical parameters such as bleeding during probing, clinical attachment loss and probing depth and bone loss that can be observed in radiology, diagnosed and recorded. (1)

*Corresponding author's ORCID ID: 0000-0001-7840-3801 DOI: https://doi.org/10.14741/ijmcr/v.6.5.14
Other advanced diagnostic techniques of periodontal disease is evaluation of host response, which includes the study of specific or non-specific mediators such as albumin, globulin and creatine phosphokinase enzyme by biochemical methods or immunological response that as part of the individual periodontal infections are known.Potential resources in these studies included samples of saliva, gingivo cervicular fluid (GCF) and serum. Regarding the relationship between periodontal disease and general health and communication conditions in other organs, there is possible relation between the disease with blood factors such as serum proteins (albumin, globulin, enzyme creatine phosphokinase, etc. $(1,2)$ 
CPK (Creatine Phospho Kinase enzyme) which also known by names creatine kinase or phosphokinase, and can be expressed in a variety of tissues and cells. This enzyme catalyzes the production of creatine phosphate, which the enzymatic reaction is also reversible (3). Some biochemical markers, including proteins changes GCF such as albumin, total protein, globulin and the activity of some enzymes, including (CPK in GCF of patients before and after treatment may be different.

Sohani et al (2015) studied several salivary enzymes in patients with moderate or severe periodontitis. This cross-sectional study via statistical tests attempted to examine the relationship between salivary enzymes such as CPK, ALT, AST, ALP and LDH levels and periodontal disease in the saliva of 14 healthy participations and 40 patients. In this study they found that there is a significant relationship between levels of salivary enzymes and periodontal disease and we can measure these enzymes in saliva, as useful markers for diagnosis, prognosis and assessment of the therapy effects in periodontal patients. (4)

Kaur $\mathrm{N}$ et al (2015) investigated the relationship between serum albumin with chronic periodontitis. In this study, serum albumin level in 60 subjects of both gender with age range 40-70 years in both healthy subjects and patient with chronic periodontitis with Attachment loss more than $5 \mathrm{~mm}$ by statistical tests performed. In this study they found that serum levels of albumin in patients with chronic periodontitis is less than healthy subjects and also there was not observed a significant relationship between chronic periodontitis and serum albumin level ( $p$ $<0.0001$ ). (5)

Ganji et al (2011) in a study investigated the relationship between cytokine concentration and $\mathrm{L}$ protein in GCF of periodontal disease. In this case-control and analytical study, GCF samples of 40 patients (20 patients with gingivitis and 20 periodontitis) were collected. ELISA technique to measure the concentration of IL-17A and RANKL in GCF samples was used. The difference between the two groups via Mann-Whitney $U$ test and correlation via Spearman correlation coefficient test were evaluated. The results of this study showed that there was no statistically significant difference between the two groups in terms of the concentration of IL-17A, so that cytokines in the group periodontitis has higher concentration, but no significant difference was observed on the RANKL. Increasing the concentration of IL-17A in periodontal disease indicate that IL-17A probably plays an important role in the disease and it can be mentioned that $\mathrm{T}$ helper 17 (TH-17) is involved in the pathogenesis of periodontal diseases in independent path than RANKL path in tissue degradation. Given the direct correlation between the concentration of IL-17A with PD and CAL in the sampling area, it assumed the involvement of IL-17A in changes of periodontal tissue destruction is strong. Of course, more extensive researches are necessary to ensure above hypotheses (6).
Larena et al (2010) in a study compare protein blood levels in patients with chronic periodontitis and healthy samples. The results of this study showed that blood concentrations of proteins (albumin, hemoglobin, immunoglobulins) among periodontal patients is higher than healthy samples (7).

Abdolsamadi et al (1389) investigated the serum albumin concentration, as a prognostic marker of severity of periodontal disease on 64 patients with chronic periodontitis and 64 healthy subjects aged 50-20 years, after analyzing the periodontal and systemic $2 \mathrm{cc}$ blood were taken from all subjects and serum albumin concentration using COBASMIRA was measured. Mean serum albumin concentration was $2.7 \pm 5.05$ in patients with chronic periodontitis and $0.39 \pm 4.40$ in healthy subjects and the difference was statistically significant. The serum albumin concentration between moderate and mild degree of illness was also significantly different. In general, serum albumin concentration in mild, moderate and severe chronic periodontitis compared to the control group. (8)

Azizi et al (2009) attempted to evaluate the concentration of albumin and total protein and relationship between periodontal parameters in patients who have received warfarin. In this case-control study participants were divided into two groups: patients who received warfarin (cases) and those not taking any medication (control group). In both groups, plaque index $(\mathrm{PI})$, gingival index $(\mathrm{GI})$, probing depth (PD), bleeding on probing (BOP) and clinical attachment loss (CAL) and total protein and albumin were investigated. The data using $T$ test and Pearson were analyzed. The results of this study indicate that the albumin in the treatment group was significantly different from the control group, although this difference was not significant in total protein of saliva (9).

Ogawa et al (2006) evaluated the relationship between periodontal disease and general health status in community-dwelling elderly. Serum albumin level was detected by the bromcresol green albumin (BCG) method and the data for serum albumin were available in 368 subjects aged 75 years. The findings of the present study indicated that there might be an inverse relationship between periodontal disease and serum albumin concentration in these elderly subjects (10).

Takashi et al (2004) were compared total protein concentrations and protein fractions among dentulous subjects, edentulous subjects and periodontitis patients. Eighty-five subjects/patients in total were studied, who consisted of 52 dentulous subjects with diagnosed normal periodontal tissue, 18 edentulous subjects using complete dentures and 15 patients with diagnosed periodontal disease. Total protein concentration was measured using dot blotting-silver staining, and protein fractions were analyzed using cellulose acetate membrane electrophoresis and silver staining. The results show total protein concentration was highest in the group of periodontal disease patients and significantly different 
from that in the group of dentulous and healthy subjects. (11)

Petrovich luA et al (1996) evaluated the activity of glutamate dehydrogenase, gamma-glutamyl transpeptidase and creatine kinase in saliva of gingivitis. The activity of glutamate dehydrogenase tends to decrease in gingivitis and diminishes in periodontitis. But gamma-glutamyl transpeptidase and creatine kinase increases in gingivitis and in periodontitis, their activity are higher than that in gingivitis. However, in this study stated that these changes are all in the mouth and after mixing with other fluids in the mouth and in salivary ducts is not visible. (12)

Huang JS et al (1990) were examined the isoenzyme patterns of creatine kinase (CK) and lactate dehydrogenase (LDH) in cultured fibroblasts in normal gingival and pathologic. Gingival fibroblasts from normal subjects and from patients with periodontitis and gingival fibroblasts as experiment group, and skin fibroblasts considered as control group.The results of this study states that isoenzymes creatine kinase-muscle in periodontitis was significantly higher than healthy gums and the lowest activity of these enzymes in the gums of patients with gingival fibromatosis was reported. (13)

\section{Methods and materials}

The present study was an analytic, prospective study which patients were randomly sampled. According to the study the relations that can be used to determine the sample size was not found and given the laboratory study, 30 patients referred to Periodontics ward of Dental School in Mashhad for treatment of moderate to severe chronic periodontitis via target based sampling for this study were considered. All the ethics principles of research, including confidentiality of participants, the right to withdraw from the study, oral and written informed consent were taken into consideration. Inclusion criteria included in Attachment loss as bags with a depth of 4-6 $\mathrm{mm}$ and if possible the same plaque control (the plaque index between patients and health education given and patients with acceptable control dental plaque, plaque index below 20\%) were selected. Exclusion criteria include periodontal abscess and preapical and history of systemic disease, smoking and...

After observing isolation by cotton rolls, 50 to $100 \mathrm{ml} \mathrm{GCF}$ via 30 paper point and wetting with a height of $5 \mathrm{~mm}$ of the area with more involvement in terms of standard of patients with moderate periodontitis pre-treatment phase 1 (scaling \& root planning) and 4 weeks after the treatment is provided. Then changes in albumin, total protein, globulin and CPK enzyme activity in the GCF using biochemical kits measured.

Four experimental methods for measuring were used; of course, GCF samples before using biochemical kits were diluted by buffer:

\section{Microalbumin test kit (measuring albumin immunoturbidometrically)}

Microalbumin test used to quantitative measurement of microalbumin in the urine. Absorbance of turbid solution produced at $340 \mathrm{~nm}$ with albumin concentration in collected samples is proportional. The microalbumin measurable range is between $500-0.25 \mathrm{mg} / \mathrm{l}$. This test was more accurate than similar tests.

\section{Biochemical diagnostic kit for urine protein}

This method based on the principle of protein reaction present in the sample by mixing red pyrogallol and molybdate and acidic compounds that combine color change is measured by spectrophotometry. This test is also more accurate than similar tests.

Diagnostic kit for enzyme CPK (quantitative detection of CPK in serum or plasma with photometric method)

Test method is as follows, patient samples $(40 \mathrm{ml})$ with distilled water $(40 \mathrm{ml})$ mixed solution 1 and 2 (reagent solution) mixed and read the optical density after 3 minutes (initial optical density), then enable chronometer and exactly determine difference of optical density after 1,2 and 3 minutes of minutes. Then sums difference of optical density after 1, 2 and 3 minutes and divided on 3 and multiplied by the obtained mean 4127 number. Then data via SPSS software version 16 and appropriate statistical tests were analyzed.

In our study, mean CPK in patients with chronic periodontitis before treatment was further after treatment but not significantly different, but the average amount of albumin, total protein and globulin chronic periodontitis patients before treatment lesser than after treatment but there was no significant difference either.

\section{Results}

This study is a clinical trial to evaluate the albumin, total protein, globulin and creatine phosphodiesterase kinase enzyme (CPK) in GCF before and after treatment of 30 patients with chronic periodontitis. In this section, albumin, total protein, globulin and creatine phosphodiesterase kinase enzyme (CPK) in GCF before and after treatment evaluated using statistical tests. Using the Kolmogorov-Smirnov test founded that data from the total protein, globulin and CPK enzyme activity are not normally distributed and only data from the albumin in GCF in patients with chronic periodontitis is normally distributed. 
Table 1: Indices of central dispersion albumin, total protein, globulin and CPK enzyme activists in GCF of patients with chronic periodontitis before and after treatment

\begin{tabular}{|c|c|c|c|c|c|}
\hline Variables & Number & Mean & Standard deviation & Minimum & Maximum \\
\hline CPK before treatment & 30 & 9.6 & 8.96 & 1 & 34 \\
\hline CPK after treatment & 30 & 9.4 & 9.53 & 3 & 28 \\
\hline Albumin before treatment & 30 & 0.01 & 0.003 & 0 & 0.03 \\
\hline Albumen after treatment & 30 & 0.11 & 0.1 & 0.03 & 0.3 \\
\hline Total protein before treatment & 30 & 0.18 & 0.11 & 0 & 0.5 \\
\hline Total protein after treatment & 30 & 0.64 & 0.29 & 0.38 & 1.2 \\
\hline Globulin before treatment & 30 & 0.17 & 0.11 & 0 & 0.5 \\
\hline Globulin after treatment & 30 & 0.52 & 0.2 & 0.3 & 0.9 \\
\hline
\end{tabular}

In the above table, central dispersion index of albumin, total protein, globulin and CPK enzyme activity in the GCF of patients with chronic periodontitis before and after treatment discussed and we observed that the mean CPK enzyme activity before treatment $8.96 \pm 9.6$ and mean CPK enzyme activity after treatment $9.53 \pm 9.4$ and the mean albumin before treatment $0.003 \pm 0.01$ and the mean albumin after treatment $0.1 \pm 0.11$ and the mean total protein level before treatment $0.11 \pm 0.18$ and mean total protein level after treatment $0.29 \pm 0.64$ and globulin before treatment $0.11 \pm 0.17$ and globulin after treatment $0.2 \pm 0.52$.

Table 2: Comparison of CPK enzyme activity in the GCF of patients with chronic periodontitis before and after treatment

\begin{tabular}{|c|c|c|c|c|}
\hline Variables & Number & Mean & $\begin{array}{c}\text { Standard } \\
\text { deviation }\end{array}$ & $\begin{array}{c}\text { Test } \\
\text { result }\end{array}$ \\
\hline $\begin{array}{c}\text { CPK before } \\
\text { treatment }\end{array}$ & 30 & 9.6 & 8.96 & \multirow{2}{*}{$\mathrm{P}=0.54$} \\
\cline { 1 - 4 } $\begin{array}{c}\text { CPK after } \\
\text { treatment }\end{array}$ & 30 & 9.4 & 9.53 & \\
\hline
\end{tabular}

In the table above, comparing the CPK enzyme activity in the GCF of patients with chronic periodontitis before and after treatment using the Wilcoxon test, founded that the CPK enzyme activity of GCF in patients with chronic periodontitis before and after treatment were not significantly different $(P=0.54)$.

Table 3: Comparison of the total protein level in GCF of patients with chronic periodontitis before and after treatment

\begin{tabular}{|c|c|c|c|c|}
\hline Variables & Number & Mean & $\begin{array}{c}\text { Standard } \\
\text { deviation }\end{array}$ & Test result \\
\hline $\begin{array}{c}\text { Total protein } \\
\text { before } \\
\text { treatment }\end{array}$ & 30 & 0.18 & 0.11 & \multirow{2}{*}{$\mathrm{P}<0.0001$} \\
\hline $\begin{array}{c}\text { Total protein } \\
\text { after treatment }\end{array}$ & 30 & 0.64 & 0.29 & \\
\hline
\end{tabular}

In the table above, comparing the total protein level in GCF of patients with chronic periodontitis before and after treatment using the Wilcoxon test, and it found that the total protein level in GCF of patients with chronic periodontitis before and after treatment were significantly different $(P<0.0001)$.
Table 4: Comparison of the globulin in the GCF of patients with chronic periodontitis before and after treatment

\begin{tabular}{|c|c|c|c|c|}
\hline Variables & Number & Mean & $\begin{array}{c}\text { Standard } \\
\text { deviation }\end{array}$ & Test result \\
\hline $\begin{array}{c}\text { Globulin before } \\
\text { treatment }\end{array}$ & 30 & 0.17 & 0.11 & \multirow{2}{*}{$\mathrm{P}<0.0001$} \\
\cline { 1 - 3 } $\begin{array}{c}\text { Globulin after } \\
\text { treatment }\end{array}$ & 30 & 0.52 & 0.2 & \\
\hline
\end{tabular}

In the table above, comparing globulin levels in GCF of patients with chronic periodontitis before and after treatment using the Wilcoxon test, it founded that the GCF of globulin in patients with chronic periodontitis before and after each treatment were significantly different $(P<0.0001)$.

Table 5: Comparison of albumin in the GCF of patients with chronic periodontitis before and after treatment

\begin{tabular}{|c|c|c|c|c|}
\hline Variables & Number & Mean & $\begin{array}{c}\text { Standard } \\
\text { deviation }\end{array}$ & $\begin{array}{c}\text { Test } \\
\text { result }\end{array}$ \\
\hline $\begin{array}{c}\text { Albumin before } \\
\text { treatment }\end{array}$ & 30 & 0.01 & 0.003 & \multirow{2}{*}{$\mathrm{P}<0.0001$} \\
\hline $\begin{array}{c}\text { Albumen after } \\
\text { treatment }\end{array}$ & 30 & 0.11 & 0.1 & \\
\hline
\end{tabular}

In the above table, comparing albumin in the GCF of patients with chronic periodontitis before and after treatment using Paired T test, it founded that the amount of albumin in the GCF of patients with chronic periodontitis before and after treatment were significantly different $(P<0.0001)$.

\section{Discussion and conclusion}

\section{Discussion}

Today, the relationship between oral diseases such as periodontitis with general health condition is considered and albumin as the main indicator of oral health is considered. (11) Since saliva and its composition could reflect the body's health status and sampling it than serum for patients is easier and more acceptable (14)

Periodontal disease is an inflammatory disease of the supporting tissues of the teeth caused by certain microorganisms and leads to progressive destruction of the periodontal ligament, alveolar bone with pocket formation or the recession, or both. Periodontal disease is 
usually based on clinical parameters such as bleeding during probing, clinical attachment loss and probing depth and bone loss that can be observed in radiology, diagnosed and recorded. (1)

Other advanced diagnostic techniques of periodontal disease is evaluation of host response, which includes the study of specific or non-specific mediators such as albumin, globulin and creatine phosphokinase enzyme by biochemical methods or immunological response that as part of the individual periodontal infections are known.Potential resources in these studies included samples of saliva, gingivo cervicular fluid (GCF) and serum. Regarding the relationship between periodontal disease and general health and communication conditions in other organs, there is possible relation between the disease with blood factors such as serum proteins (albumin, globulin, enzyme creatine phosphokinase, etc. $(1,2)$

CPK (Creatine Phospho Kinase enzyme) which also known by names creatine kinase or phosphokinase, and can be expressed in a variety of tissues and cells. This enzyme catalyzes the production of creatine phosphate, which the enzymatic reaction is also reversible (3). Some biochemical markers, including proteins changes GCF such as albumin, total protein, globulin and the activity of some enzymes, including (CPK in GCF of patients before and after treatment may be different.

Sohani et al (2015) studied several salivary enzymes in patients with moderate or severe periodontitis. This cross-sectional study via statistical tests attempted to examine the relationship between salivary enzymes such as CPK, ALT, AST, ALP and LDH levels and periodontal disease in the saliva of 14 healthy participations and 40 patients. In this study they found that there is a significant relationship between levels of salivary enzymes and periodontal disease and we can measure these enzymes in saliva, as useful markers for diagnosis, prognosis and assessment of the therapy effects in periodontal patients. (4)

Petrovich luA et al (1996) evaluated the activity of glutamate dehydrogenase, gamma-glutamyl transpeptidase and creatine kinase in saliva of gingivitis. The activity of glutamate dehydrogenase tends to decrease in gingivitis and diminishes in periodontitis. But gamma-glutamyl transpeptidase and creatine kinase increases in gingivitis and in periodontitis, their activity are higher than that in gingivitis. However, in this study stated that these changes are all in the mouth and after mixing with other fluids in the mouth and in salivary ducts is not visible. (12)

Huang JS et al (1990) were examined the isoenzyme patterns of creatine kinase (CK) and lactate dehydrogenase (LDH) in cultured fibroblasts in normal gingival and pathologic. Gingival fibroblasts from normal subjects and from patients with periodontitis and gingival fibroblasts as experiment group, and skin fibroblasts considered as control group.The results of this study states that isoenzymes Creatine kinase-muscle in periodontitis was significantly higher than healthy gums and the lowest activity of these enzymes in the gums of patients with gingival fibromatosis was reported. (13)

In our study, the activity of the enzyme creatine phosphate kinase in chronic periodontitis patients before treatment is (mean $=9.6$ ) than after treatment (mean = 9.4) but unlike previous studies, this difference was not significant $(P=0.54)$. Although this relationship is not statistically significant, it is also probably due to low sample size.

Kaur $\mathrm{N}$ et al (2015) investigated the relationship between serum albumin with chronic periodontitis. In this study, serum albumin level in 60 subjects of both gender with age range 40-70 years in both healthy subjects and patient with chronic periodontitis with Attachment loss more than $5 \mathrm{~mm}$ by statistical tests performed. In this study they found that serum levels of albumin in patients with chronic periodontitis is less than healthy subjects and also there was not observed a significant relationship between chronic periodontitis and serum albumin level ( $p<0.0001)$. (5)

Larena et al (2010) in a study compare protein blood levels in patients with chronic periodontitis and healthy samples. The results of this study showed that blood concentrations of proteins (albumin, hemoglobin, immunoglobulins) among periodontal patients is higher than healthy samples (7).

Abdolsamadi et al (1389) investigated the serum albumin concentration, as a prognostic marker of severity of periodontal disease on 64 patients with chronic periodontitis and 64 healthy subjects aged $50-20$ years, after analyzing the periodontal and systemic $2 \mathrm{cc}$ blood were taken from all subjects and serum albumin concentration using COBASMIRA was measured. Mean serum albumin concentration was $2.7 \pm 5.05$ in patients with chronic periodontitis and $0.39 \pm 4.40$ in healthy subjects and the difference was statistically significant. The serum albumin concentration between moderate and mild degree of illness was also significantly different. In general, serum albumin concentration in mild, moderate and severe chronic periodontitis compared to the control group. (8)

Ogawa et al (2006) evaluated the relationship between periodontal disease and general health status in community-dwelling elderly. Serum albumin level was detected by the bromcresol green albumin (BCG) method and the data for serum albumin were available in 368 subjects aged 75 years. The findings of the present study indicated that there might be an inverse relationship between periodontal disease and serum albumin concentration in these elderly subjects (10).

In this study, like study by Kaur N, Abdolsamadi and Ogawa and unlike Larena albumin in patients with chronic periodontitis before treatment (mean $=0.01 \pm 0.003$ ) was less than the albumin in patients after treatment (mean=0.11 \pm 0.1 ) and albumin before and after treatment were significantly different $(P<0.0001)$.

Takashi et al (2004) were compared total protein concentrations and protein fractions among dentulous 
subjects, edentulous subjects and periodontitis patients. Eighty-five subjects/patients in total were studied, who consisted of 52 dentulous subjects with diagnosed normal periodontal tissue, 18 edentulous subjects using complete dentures and 15 patients with diagnosed periodontal disease. Total protein concentration was measured using dot blotting-silver staining, and protein fractions were analyzed using cellulose acetate membrane electrophoresis and silver staining. The results show total protein concentration was highest in the group of periodontal disease patients and significantly different from that in the group of dentulous and healthy subjects. (11)

In our study, unlike the study Takashi total protein in patients with periodontitis before treatment (mean $=$ $0.18 \pm 0.11$ ) was less than total protein in patients after treatment $($ mean $=0.64 \pm 0.29)$ and were significantly different $(P<0.0001)$.

Total protein after treatment increased due to proteins such as matrix metalloproteinases and other items that reduce their role in the pathogenesis of periodontitis are proven. Further studies needed to investigate in this field. The difference of results with other studies may be due to sample size or sampling time after treatment.

Ganji et al (2011) in a study investigated the relationship between cytokine concentration and $L$ protein in GCF of periodontal disease. In this case-control and analytical study, GCF samples of 40 patients (20 patients with gingivitis and 20 periodontitis) were collected. ELISA technique to measure the concentration of IL-17A and RANKL in GCF samples was used. The difference between the two groups via Mann-Whitney $U$ test and correlation via Spearman correlation coefficient test were evaluated. The results of this study showed that there was no statistically significant difference between the two groups in terms of the concentration of IL-17A, so that cytokines in the group periodontitis has higher concentration, but no significant difference was observed on the RANKL. Increasing the concentration of IL-17A in periodontal disease indicate that IL-17A probably plays an important role in the disease and it can be mentioned that Thelper 17 (TH-17) is involved in the pathogenesis of periodontal diseases in independent path than RANKL path in tissue degradation. Given the direct correlation between the concentration of IL-17A with PD and CAL in the sampling area, it assumed the involvement of IL-17A in changes of periodontal tissue destruction is strong. Of course, more extensive researches are necessary to ensure above hypotheses (6).

In this study, unlike Ganji and Larena, globulin in patients with chronic periodontitis before treatment (mean $=0.17 \pm 0.11$ ) was lower than globulin in patients after treatment (mean $=0.52 \pm 0.2$ ) and were significantly different $(P<0.0001)$.

\section{Conclusion}

In this study, mean CPK in patients with chronic periodontitis before treatment was further after treatment but not significantly different, but the average amount of albumin, total protein and globulin chronic periodontitis patients before treatment lesser than after treatment but there was no significant difference either. It seems that in future studies to precise comparison of different enzymes with periodontal disease is required larger sample size used. In the future, it is better described studies in this area rather than just comparing two groups of patients with periodontitis and healthy sample. The comparison of different enzymes between different severity of periodontal disease and various types of this disease in term of unknown aspect of this field requires further and wider study.

\section{References}

[1]. Newman MG, Takei HH, Carranza FA. Carenza's Clinical Periodontatology. 11th ed. 2011. Philadelphia: WB Saunders Co; 104-6, 506-511.

[2]. Koss MA, and etc. Changes in saliva protein composition in patients with periodontal disease. Acta Odontol Latinoam. 2009; 22(2): 105-12.

[3]. Nomura Y, Tamaki Y, Tanaka T, Arakawa H, Tsurumoto A, Kirimura $\mathrm{K}$, et al. Screening of periodontitis withsalivary enzyme test. J oral sci. 2006 Dec; 48(4): 177-83.

[4]. Sohani M, Saeid Akbari Taher, Nasrin Dashti, Boani, M. Zare Nahid Einollahi. To evaluate the activity of several enzymes in the saliva of patients with moderate or severe periodontitis: a pilot study. Journal of Isfahan Dental School of Medicine. 2015. 11 (2). 170-179

[5]. Kaur N, Kaur N, Sarangal V. A study to evaluate the correlation of serum albumin levels with chronic periodontitis. Indian J Dent Res. 2015 Jan-Feb;26(1):11-4.

[6]. Ganji A., Amini Behbahani A, Satari Moseh, Baqir, Mehdi Samani. The relationship between cytokine concentration and $L$ protein found in GCF and periodontal diseases martyr Beheshti University Dental Journal, Volume 29, Number 1 Spring 2011, 22-28

[7]. Larena D, and etc. Comparative proteomic analysisof whole saliva from chronic periodontitis patients JOURN ALOFPROTEOMICS73(2010) 1334-1341.

[8]. Abdulsamadi Hamid, Hamid Mortazavi, Saghafi Sh, Khoshhal M., Mahnaz Afshar, Jamshidi Z. Evaluation of serum albumin in chronic periodontitis. Journal of Dental School, Mashhad, 1389, Volume 34, Issue 3. 96-189.

[9]. Azizi A, Lawaf Sh. The Assessment of Periodontal Parameters, Salivatory Total Protein and Albumin Contents in Patients Taking Warfarin. Shiraz Univ Dent J 2010; Vol. 10, Supplement.

[10]. Ogawa H, Yoshihara A, Amaraswna N, Hirotomi T, Miyazaki H. Association between serum albumin and periodontal disease in community-dwelling elderly. J Clin Periodontol. 2006 May; 33(5): 312-6.

[11]. Takahashi Y, Shiba A, Shiba K. Differences in whole salivary total protein concentration and protein fractions among the groups of dentulous subjects, edentulous subjects and periodontitis patients. Nihon Hostetsu Shika Gakkai Zasshi 2004.12; 48(5): 723-32.

[12]. Petrovich, luA, et al. "[Activity of glutamate dehydrogenase, gamma-glutamyltranspeptidase and creatine kinase in saliva in gingivitis]." Patologicheskaia fiziologiia i eksperimental'naia terapiia 4 (1995): 28-30

[13]. Huang, J. S., T. Z. Liu, and R. S. Bhatnagar. "Creatine kinase and lactate dehydrogenase isoenzyme patterns in cultured normal and pathological gingival fibroblasts." Journal of the Formosan Medical Association= Taiwan yi zhi 89.2 (1990): 126-131.

[14]. Streckfus CF, Bigler LR. Saliva as a diagnostic fluid. Oral Dis 2002;8(2):69-76. 